\title{
DE CÓMO KANT TRATARÍA LA EQUIDAD DE GÉNERO
}

Bryan Benjamín Gastelum García ${ }^{1}$

Me dormí y soñé que la vida era belleza;

me desperté y vi que era deber.

-Immanuel Kant.

Sumario: I. Introducción. II. Objetivo. III. Kant y la equidad de género. IV. Discusión. V. Conclusiones.

\section{Resumen}

El filósofo Immanuel Kant fue un erudito de la llustración de gran prestigio que llegó a concebir un sistema filosófico de gran relevancia, donde muchas de sus ideas tales como el obrar y la libertad humana son discutidas hoy en día. El ideal que pregonaba la época de la Ilustración no representaba precisamente la igualdad hacia la mujer, así como en la filosofía kantiana se difiere de ello.Sin embargo, fue muy particular la concepción que tuvo el filósofo alemán sobre la igualdad y la equidad entre individuos, así como el "género" que mantiene cierta controversia hoy en día.

Para comprender el tema de la equidad de género en la actualidad y contrastar con la postura al respecto de Immanuel Kant, acudiremos a sus obras importantes como la Crítica de la razón pura, y Fundamentación de la metafísica de las costumbres.

De esta manera tendremos un nuevo referente para el ejercicio de políticas encaminadas a disminuir el problema de violencia que afecta tanto al hombre como a la mujer.

Palabras clave: Género, equidad, libertad, Immanuel Kant, violencia.

\footnotetext{
${ }^{1}$ Estudiante del Séptimo Semestre de la Licenciatura en Derecho de la Universidad de Sonora, e-mail: totodx189@gmail.com
} 


\section{De cómo Kant trataría la Equidad de Género \\ Año 11, Número 20 enero-junio 2019 Bryan Benjamín Gastelum García}

\section{Abstract}

The philosopher Immanuel Kant was a scholar of the prestigious Enlightenment who came to conceive of a philosophical system of great relevance, where many of his ideas such as work and human freedom are discussed today.

The ideal proclaimed by the Enlightenment period did not represent equality towards women, as it differs from this in Kantian philosophy.

However, the German philosopher's conception of equality and equity between individuals, as well as the controversial "gender", was very particular.

To understand the issue of gender equity today and contrast with Immanuel Kant's position on the subject, we will look at his important works such as Critique of Pure Reason and Rationale for Metaphysics of Habits.

In this way, we will have a new benchmark for the exercise of policies aimed at addressing the problem of violence affecting both men and women.

Key words: Gender, equity, freedom, Immanuel Kant, violence.

\section{INTRODUCCIÓN}

Es indiscutible que el pensamiento que preponderaba en la época de la revolución francesa (aun cuando existía un trasiego de opiniones) es notablemente diferente al siglo XXI. Sin embargo, muchas de las ideas que representaban el espíritu de la época revolucionaria en Europa del siglo XVIII, tuvieron gran impacto, al grado de ser la base para la constitución de diversos sistemas jurídicos que han evolucionado, y ahora rigen actualmente en distintas naciones.

Siguiendo este orden de ideas, los eventos de la llustración culminaron en uno de los mayores logros que ha presenciado la humanidad en materia política y progreso social, es decir, la formación y establecimiento de la Declaración Universal de los Derechos humanos; declaración adoptada por la Asamblea General de las Naciones Unidas, el 10 de diciembre de 1948, junto a los Pactos 
Internacionales de Derechos humanos y diversos protocolos, constituyéndose de manera onerosa la Carta Internacional de los Derechos humanos.

Lo anterior quiere decir que se consagra a través de una normativa internacional "la libertad e igualdad en dignidad y derechos..." de todos los seres humanos, sin excluir su etnicidad, color, sexo, o cualquier otra condición. ${ }^{2}$

Pero uno de los principales problemas que atentan contra los Derechos Humanos es sin duda alguna la falta de equidad de género: mecanismo social que provoca la violencia hacia la mujer.

Con respecto a lo anterior, hablar sobre la equidad de género como menciona la doctora Adria González (2010) "implica recordar que desde sus inicios la historia de la humanidad ha estado sellada profundamente por el patriarcado". 3

Esto quiere decir que el papel desempeñado por la mujer durante generaciones en la esfera civil y jurídica se ha visto opacado por el hombre, vulnerando principalmente la capacidad y dignidad de su persona.

\section{OBJETIVO}

Realizaremos un análisis sobre la "equidad de género", desentrañando este término y esclareciendo sus elementos, apoyados por el sistema filosófico de Immanuel Kant.

\footnotetext{
2 Universal Declaration Of Human Rights, "Carta Internacional de los Derechos Humanos,"

http://web.archive.org/web/20170831154642/http://www.ohchr.org/Document s/Publications/FactSheet2Rev.1en.pdf (En inglés). 3 González Beltrones, Adria Velia, "Equidad de Género en las Instituciones Educativas," BIOLEX. Revista Jurídica del Departamento de Derecho. Academia de Derecho Administrativo. Tercera Época Año2. No. 3 / JulioDiciembre, 2010, http://www.biolex.uson.mx/revistas/articulos/3-art02.pdf Consultado el 12 de junio de 2018
} 


De cómo Kant trataría la Equidad de Género
Año 11, Número 20 enero-junio $2019 \quad$ Bryan Benjamín Gastelum García

\section{KANT Y LA EQUIDAD DE GÉNERO}

\section{- La equidad:}

¿Cómo podemos concebir la equidad bajo la perspectiva Kantiana exactamente?

En la obra Principios Metafísicos del derecho, Immanuel Kant expresa que todo derecho en un sentido estricto va acompañado por una ley jurídica que puede obligar a exigir ese legitimo ordenamiento, pero se puede concebir otro derecho en sentido lato, del cual una ley no puede determinar la facultad de obligar. Esta "normatividad" que carece de atribuciones imperativas, se encuentra de dos modos: equidad y derecho de necesidad; donde encuadra a la Equidad bajo el sentido lato de la siguiente forma:

La equidad (objetivamente considerada) no es un título en cuya virtud se pueda compeler a otro al cumplimiento de un deber puramente moral (ser benévolo o benéfico). El que pide algo en nombre de este principio se funda en su derecho; [...] Pero este mal [no tener la facultad de obligar] no puede corregirse por medio del derecho, por más que se refiere a una cuestión de derecho, porque la reclamación que se funda en la equidad no tiene fuerza más que en el tribunal de la conciencia (forum cceli), al paso que la cuestión de derecho se discute en el tribunal civil (forum soli s. civile). ${ }^{4}$

Un ejemplo de esto, podría ser el problema del tabaquismo. Hasta 1981 se publicó por primera vez un estudio en Japón que siguió durante 14 años en

${ }^{4}$ Kant, Immanuel, Principios metafísicos de la doctrina del derecho, Universidad Nacional Autónoma de México, Dirección general de publicaciones, México, 1968, pp.49 
la región a 91,540 esposas no fumadoras mayores de 40 años. En dicho estudio se presentó el resultado de un exceso de mortalidad, debido a las altas tasas cáncer de pulmón ocasionado por la exposición nociva de los hábitos de sus esposos fumadores. ${ }^{5}$

Respecto a lo anterior, sin mencionar los cientos de estudios que se han realizado sobre el consumo del tabaco y el riesgo que provoca el humo en los fumadores tanto activos como pasivos, debemos tener presente el fundamento de la Constitución Mexicana al mencionar en el art. $4^{\circ}$, párrafo $3^{\circ}$, que "Toda persona tiene derecho a la protección de la salud".

Siguiendo este planteamiento, la Ley General para el Control del Tabaco, en el Capítulo III, artículo 27, expresa la disposición reglamentaria para consumir productos de tabaco en dos tipos de zonas exclusivas:

1) Espacios al "aire libre";

2) Espacios internos aislados con mecanismos para evitar la propagación de partículas a espacios $100 \%$ libre de tabaco.

Sobre esta situación, a pesar de los esfuerzos legislativos para generar conciencia entre la población debido a los efectos nocivos del cigarro, así como los daños que puede provocar el humo en personas no fumadoras, es común ver en las calles, parques y plazas a gente fumar en medio de personas que no lo hacen.

Si bien, el Estado tutela el derecho a la salud a través de leyes y reglamentos, tomando el caso anterior, no se le puede exigir a una autoridad

5 Takeshi Hirayama., "Non-smoking wives of heavy smokers have a higher risk of lung cancer: a study from Japan," British Medical Journal Clinic, volume 282, 1981, pp.183[en línea] Disponible en:

https://www.bmj.com/content/bmj/282/6259/183.full.pdf ; Consultado el 26 de agosto de 2018 


\section{De cómo Kant trataría la Equidad de Género \\ Año 11, Número 20 enero-junio 2019 Bryan Benjamín Gastelum García}

cercana (tómese de ejemplo un policía municipal) que ordene a un fumador cualquiera a apagar su cigarrillo si se encuentra al aire libre. ${ }^{6}$

Esta reclamación no tiene más fuerza que en el tribunal de la conciencia del fumador, dado que la persona no puede alegar el desconocimiento de los efectos nocivos que puede provocar el consumo de nicotina, así como sus consecuencias adversas provocadas por el humo del cigarro en las personas cercanas a su alrededor, puesto que la publicidad sobre los peligros de fumar y lo que puede provocar en las personas pasivas se exhibe en los mismos productos de venta y demás medios de difusión constantemente.

Con lo anterior se expresa que la concepción de Kant sobre la "equidad" es una idea que se sitúa en una esfera Metajurídica, pues no concierne directamente a las leyes externas que regulan a los individuos de una sociedad, sino más bien, pertenece al inmanente fuero de la conciencia.

Si tomamos en cuenta uno de los principios expuestos en la Fundamentación de la metafísica de las costumbres: "Obra sólo como si la máxima de tu acción fuera a tornarse por tu voluntad en ley universal", ${ }^{7}$ el postulado kantiano sobre equidad se erige como ley moral en la manera de obrar.

En el caso de que un individuo obra con otro de manera que no sea injuriado, pues espera el mismo trato recíproco, el individuo no puede obligar (basándonos en el principio de equidad) al otro, a ejercer el mismo trato.

Verbigracia, podemos citar a aquel individuo que hace uso de un inmueble familiar prometiendo el pago pronto sin contrato alguno. Sin embargo, al cabo

6 Podría actuar en casos de excepción si se encuentran cerca niños, adolescentes y grupos vulnerables. Ley General para el Control del Tabaco, Capitulo II, Artículo 9

${ }^{7}$ Kant Immanuel, Fundamentación para una metafísica de las costumbres.

España, Alianza Editorial, 2012, pp.35 
del tiempo el arrendatario se niega a pagar, mientras que el arrendador por buena fe no tiene contrato ni testigos que avalen el acuerdo.

Esto es más que una noción, pues en la realidad jurídica, la legislación mediante la etapa de investigación con el análisis de diferentes medios de prueba puede obligar a resarcir el daño a un individuo que ha causado perjuicio en contra de persona alguna si resulta culpable.

Pero esta noción ideada por Kant es una noción referida a la naturaleza humana que no se ve coaccionada en el momento de los casos de mayor necesidad de observancia judicial. Basta como ejemplo la situación de la violencia contra la mujer en todos sus aspectos, que se da mayoritariamente dentro de los hogares y lugares privados.

\section{- El género}

Dejando de momento la noción de equidad, es menester preguntar qué es el género en la condición humana. Según la SCJN, el "Género" se refiere a los atributos que, social, histórica, cultural y geográficamente, se le han asignado a los hombres y a las mujeres.

"Género" se utiliza para referirse a las características [estereotipos] que social y culturalmente se consideran identificadas como "masculinas" y "femeninas". 8 Por su contraparte, Kant define el género como:

"Concepto natural dotado de unas propiedades que podemos atribuir a cada uno de los individuos pertenecientes al mismo, pero sabiendo que las mismas se dicen de cada individuo analógicamente

${ }^{8}$ Suprema Corte de Justicia de la Nación., Protocolo de Actuación para quienes imparten justicia en casos que involucren la orientación sexual o la identidad de Género, México, 2011,pp. 12 -13 


\section{De cómo Kant trataría la Equidad de Género \\ Año 11, Número 20 enero-junio 2019 Bryan Benjamín Gastelum García}

sin que podamos nunca llegar a tener una intuición de ellas, dado que, aunque las podamos considerar reales, no quiere decir que sean sensibles y por eso necesitan ser fundadas mediante un riguroso proceso de deducción de las mismas a partir del origen mismo del género". ${ }^{9}$

Si bien, la definición ontológica que propone Kant del género parte de un simbolismo biológico, no desmerita el hecho que el concepto mismo se torna complejo al suscitar que su estructura va más allá del producto de una intuición empírica.

Al analizar la definición que otorga la SCJN, es inteligible el esfuerzo sistemático por interpretar el género. El psicólogo Díaz-Guerrero (1991) menciona que: "una actividad es sistemática cuando trata o parece tratar de abarcar todos los factores que intervienen en la producción de un fenómeno y, además, enumera reglas o principios que relacionan esos factores". ${ }^{10}$

Una de las cuestiones en la actualidad radica, verbigracia, cuando una persona se identifica sin género; ${ }^{11}$ aunque es sostenido por algunas posturas como el tercer sexo o genderqueer (tercer género).

${ }^{9}$ Cirilo Flórez Miguel, Génesis de la razón histórica, Universidad de Salamanca,España, 1983,pp. 40-41.

${ }^{10}$ Díaz-Guerrero, Rogelio, Introducción a la psicología: un enfoque sistemático México, Editorial Trillas, 1991

${ }^{11}$ Vide:Periódico ABC BITÁCORAS, El polémico caso del primer bebé sin género definido del mundo,2017[en línea]Disponible en:

http://www.abc.es/recreo/abci-polemico-caso-primer-bebe-sin-generodefinido-mundo-201707051046_noticia.html,consultado el 26 de agosto de 2018 
Esta cuestión se puede trasladar al mundo lógico-matemático bajo el axioma de regularidad (teoría de conjuntos) donde se señala que un conjunto que se integra así mismo como elemento, no puede existir.

Es decir, el "género" de la Suprema Corte se considera un conjunto de elementos pues es caracterizado por distintas variables, en este caso, atributos históricos, culturales y geográficos asignados al hombre y la mujer.

Mientras que el "sin género" al no tener ningún atributo que asignar, se considera a sí mismo como parte de su conjunto, y esto contradice el axioma de regularidad que atribuye a un conjunto una existencia independiente por la serie de elementos diferentes dentro de su contenido.

Para un mejor entendimiento, se presenta una formula y su explicación: $\forall$ : Para todos los elementos de...

A: "Sin género".

$\mathrm{n}$ : Conjunto que contiene todos aquellos elementos que mantienen en común la intersección de dos variables.

$\{A\}:$ Conjunto de "sin género".

$\emptyset$ : Conjunto vacío.

$$
\forall \boldsymbol{A} \cap\{\boldsymbol{A}\}=\{\boldsymbol{A}\}=\varnothing
$$

Para tener carácter jurídico, el "sin género" debe estar integrado por distintos elementos para cumplir con la regularidad. El elemento $\mathbf{A}$ se vuelve conjunto $\{\mathbf{A}\}$ para ser determinado como ente jurídico, y por la regularidad, $\{\mathbf{A}\}$ debe contener un elemento distinto de $\{\mathbf{A}\}$. El único elemento de $\{\mathbf{A}\}$ es $\mathbf{A}$ lo que se sigue que $\forall A \cap\{A\}$ (todos los elementos del "sin género" tienen en común los elementos del conjunto de "sin género"). 


\section{De cómo Kant trataría la Equidad de Género \\ Año 11, Número 20 enero-junio 2019 Bryan Benjamín Gastelum García}

Por lo tanto $\{\mathbf{A}\}=\emptyset$ (el conjunto de "sin género" es igual a un conjunto vacío), es decir, resulta ilógico no identificarse con algún género, pues la correlación del término responde en ultima instancia a la concepción biológica.

Lo anterior quiere decir que la fórmula de la teoría de conjuntos declara dentro de nuestro sistema de regularidad, que no existe formalmente para el ámbito de aplicación jurídica las personas "sin género". Debe tenerse en cuenta que este término utilizado por la Suprema Corte sólo distingue entre el sexo femenino y masculino, más no contempla otro género, o a alguien que carezca del mismo. ${ }^{12}$

En ese aspecto, cuando se habla de violencia de género, ¿qué nuevas medidas legislativas habrán de impulsarse para resolver los problemas jurídicos sin género?

El caso no responde a contener un problema semántico. Se debe llegar a preguntar por qué es necesario indicar que se trata de género. Puesto que cualquier tipo de violencia, en cualquiera de sus formas, es aplicado en mayor o menor grado a un ser.

Posiblemente la única diferencia reside en que los animales sufren otro tipo de violencia, por no poder ser asimilados "culturalmente" de momento, como personas. En el ejemplo del feminicidio, la víctima es asesinada por una

${ }_{12}$ La ONU reconoce que existen más géneros, pues la identidad de género no corresponde "necesariamente" al sexo biológicamente asignado al nacer, Vide: UN HUMAN RIGHTS UNHR, Statement by Vitit Muntarbhorn, Independent Expert on sexual orientation and gender identity, at the European Governmental LGBTI Focal Point Network Roundtable, Council of Europe, 2016 [en línea]Disponible en:

https://www.ohchr.org/EN/NewsEvents/Pages/DisplayNews.aspx?NewsID=2 0912\&LangID=E; consultado el 26 de agosto de 2018 
serie de factores tales como el sentimiento de odio hacia la mujer (porcausas de género).

Kant comprende el sentimiento como: "Capacidad de placer o disgusto que se experimenta con la idea de una cosa y no contiene más que lo subjetivo puro en su relación con nuestra representación". ${ }^{13}$

Para ello aclara que lo subjetivo puro de nuestras representaciones es primero, la representación: un objeto (significado), una cosa que no piensa sino por su medio.

Cuando se menciona el asesinato de una mujer por cuestiones de género (feminicidio), bajo la cosmovisión de Kant, no precisamente se refiere a la noción de un odio "racional" hacia las propiedades culturales y sociales que caracterizan al género femenino.

Más bien, se refiere a una trasgresión fundada por una experiencia individual, donde en efecto, intervienen ciertas pautas, ciertas construcciones, pero mentales que caracterizan a una persona.

\section{Discusión}

Según IFAD ${ }^{14}$, la equidad de género se puede definir como:

"La imparcialidad en el trato que reciben mujeres y hombres de acuerdo con sus necesidades respectivas ya sea con un trato igualitario o con uno diferenciado pero que se considera equivalente en lo que se refiere a los derechos, los beneficios, las obligaciones y las posibilidades. En el ámbito del desarrollo, un objetivo de equidad de género a menudo requiere incorporar

\footnotetext{
${ }^{13}$ Kant Immanuel,op.cit. Principios metafísicos...pp. 14

14 Por sus siglas en inglés International Fund Agricultural Development, Fondo Internacional para el Desarrollo Rural,
} 


\section{De cómo Kant trataría la Equidad de Género \\ Año 11, Número 20 enero-junio 2019 Bryan Benjamín Gastelum García}

medidas encaminadas a compensar las desventajas históricas y sociales que arrastran las mujeres". ${ }^{15}$

Este término tenido en cuenta como criterio técnico que se ha venido introduciendo en las legislaciones para la toma de decisiones públicas, se discierne en la terminología de Kant, pues él podría definirlo como: Principio de bondad que se erige como máxima en el obrar respecto a las condiciones y circunstancias que caracterizan a un individuo, sin compeler una retribución.

\section{CONCLUSIONES}

1. Podemos considerar los principios morales que expresa Kant no como leyes aplicables a todos los casos, sino, como una guía para resolver nuestros conflictos, y crear conciencia para un mutuo respeto:

\section{a) Universalización}

Obra de tal manera, que puedas querer al mismo tiempo que tu acción se vuelva ley universal.

b) Humanidad como un fin en sí misma

Trata a las personas como un fin y nunca como un medio.

c) Reino de los fines

Actúa de tal forma que tus acciones sean aceptadas como leyes.

d) Universal del Derecho

Es justa toda acción que no sea un obstáculo a la libertad de todos.

2. Pese a nuestras diferencias biológicas, no debemos tampoco obstinarnos a la simple idea de considerar analógicamente las distinciones entre el hombre y la mujer como menciona el filósofo

15 IFAD,Policy on gender equality and women's empowerment,2011[en línea]Disponible en: https://webapps.ifad.org/members/ec/70/docs/EC-201170-W-P-6-Rev-1.pdf ; consultado el 26 de agosto de 2018 
alemán, ya que es necesaria una rigurosa deducción. Y a partir de ello no bastarán sólo los aspectos corporales, históricos y sociales; también los aspectos mentales.

3. Podemos cuestionar nuestro margen jurídico pues en México no existe el estado de Derecho para resolver de mejor manera las controversias que se compadecen hoy en día respecto a la desigualdad y la violencia de género.

4. Kant trataría posiblemente la equidad de género como: Principio de bondad que se erige como máxima en el obrar respecto a las condiciones y circunstancias que caracterizan a un individuo, sin compeler una retribución.

\section{BIBLIOGRAFÍA}

CIRILO FLÓREZ Miguel, Génesis de la razón histórica,Universidad de Salamanca,España, 1983

DÍAZ-GUERRERO Rogelio, Introducción a la psicología: un enfoque sistemático, Editorial Trillas, México,1991

GONZÁLEZ BELTRONES Adria Velia, "Equidad de Género en las Instituciones Educativas," BIOLEX. Revista Jurídica del Departamento de Derecho. Academia de Derecho Administrativo. Tercera Época Año2. No. 3 / Julio-Diciembre (2010), http://www.biolex.uson.mx/revistas/articulos/3-art02.pdf

KANT Immanuel, Principios metafísicos de la doctrina del derecho (México: Universidad Nacional Autónoma de México, Dirección general de publicaciones, 1968).

Crítica de la razón pura (Ciudad de México, Editorial Colofón. Ciudad de México., 2017).

Fundamentación para una metafísica de las costumbres. (España: Alianza Editorial, 2012). 


\section{De cómo Kant trataría la Equidad de Género \\ Año 11, Número 20 enero-junio 2019 Bryan Benjamín Gastelum García}

SUPREMA CORTE DE JUSTICIA DE LA NACIÓN, Protocolo de Actuación para quienes imparten justicia en casos que involucren la orientación sexual o la identidad de Género, Ed. SCJN, México, 2014

\section{Legislación:}

1) Constitución De Los Estados Unidos Mexicanos.

2) Ley General Para El Control Del Tabaco.

\section{Webgrafía:}

IFAD,Policy on gender equality and women's empowerment,2011[en línea]Disponible en: https://webapps.ifad.org/members/ec/70/docs/EC2011-70-W-P-6-Rev-1.pdf

Periódico ABC BITÁCORAS, El polémico caso del primer bebé sin género definido del mundo,2017[en linea] Disponible en:

http://www.abc.es/recreo/abci-polemico-caso-primer-bebe-sin-generodefinido-mundo-201707051046_noticia.html

THE INTERNATIONAL BILL OF HUMAN RIGHTS Carta Internacional de los DerechosHumanos,2018[en linea] Disponible en:

http://web.archive.org/web/20170831154642/http://www.ohchr.org/Document s/Publications/FactSheet2Rev.1en.pdf

UN HUMAN RIGHTS UNHR, Statement by Vitit Muntarbhorn, Independent Expert on sexual orientation and gender identity, at the European Governmental LGBTI Focal Point Network Roundtable, Council of Europe, 2016 [en línea]Disponible en:

https://www.ohchr.org/EN/NewsEvents/Pages/DisplayNews.aspx?NewsID=2 0912\&LangID=E 